\title{
Incidencia de sepsis precoz por Streptococcus agalactiae en recién nacidos del Centro Hospitalario Pereira Rossell en el período 2007-2015
}

\begin{abstract}
Tamara I. Herrera', Micaela Murillo', Juan Pablo Gesuele', Mario Moraes', María Inés Mota², Claudia Gutiérrez², Adriana Varela² y Gabriela Algorta²
\end{abstract}

\footnotetext{
Centro Hospitalario Pereira Rossell, Montevideo, Uruguay 'Servicio de Recién Nacidos. Departamento de Neonatología. ${ }^{2}$ Departamento de Bacteriología.

Los autores declaran no tener conflicto de interés. No hubo fuente de financiamiento externa.

Recibido: 4 de julio de 2017 Aceptado: 25 de mayo de 2018

Correspondencia a: Tamara I. Herrera tamaraherrera143@gmail.com
}

\section{Incidence of group B streptococcal early-onset sepsis in infants born at Centro Hospitalario Pereira Rossell between 2007-2015}

Background: Group B Streptococcus (GBS) disease remains the leading cause of early-onset sepsis (EOS) in developed countries despite effective prophylaxis strategies. Aims: To describe the incidence, clinical features and mortality of GBS EOS in infants born at Centro Hospitalario Pereira Rossell (CHPR) and analyse failure of adherence to prevention strategies. Methods: Retrospective review of EOS cases between 2007 and 2015 collected from the bacteriology laboratory database. Results: Fifteen cases of GBS EOS were identified, with an incidence of $0.23 \%$ during the study period. Intrapartum antibiotic prophylaxis (IAP) was not administered in any of the cases. All infants were symptomatic within the first 15 hours of life, mainly due to respiratory signs $(80 \%)$. In one case, GBS was isolated from spinal fluid. Mortality rate was 20\%. All deaths occurred in the first 24 hours of life, corresponding two thirds to preterm infants. Conclusion: The incidence of GBS EOS at CHPR was similar to other centers where IAP is implemented. Better adherence to prophylaxis strategies could reduce the incidence.

Key words: Neonatal sepsis; Streptococcus agalactiae; group B Streptococcus; intrapartum antibiotic prophylaxis.

Palabras clave: Sepsis neonatal; Streptococcus agalactiae; Streptococcus grupo B; quimioprofilaxis intraparto.

\section{Introducción}

$\mathrm{L}$ a sepsis neonatal continúa siendo una complicación frecuente con elevada morbimortalidad en el período neonatal. Los mecanismos de inmunidad de los recién nacidos, y particularmente de los prematuros, son relativamente deficientes debido a inmadurez del sistema inmune, así como a una disminución del pasaje transplacentario de anticuerpos maternos, todo lo que contribuye a la vulnerabilidad a infecciones graves bacterianas, fúngicas y virales ${ }^{1}$. El diagnóstico de sepsis neonatal representa un gran desafío ya que su presentación es inespecífica y la utilidad de las pruebas de laboratorio actuales es limitada debido a su baja sensibilidad ${ }^{2,3}$. El aislamiento del microorganismo a partir de sangre $u$ otros líquidos estériles es considerado el estándar de oro para el diagnóstico de sepsis neonatal ${ }^{1}$.

La mayoría de los estudios epidemiológicos y ensayos clínicos definen sepsis neonatal precoz como aquella que ocurre en las primeras $72 \mathrm{~h}$ de vida y es causada por transmisión vertical de los microorganismos involucra$\operatorname{dos}^{4-6}$. Streptococcus agalactiae (Streptococcus del grupo B-SGB) es una de las causas más frecuentes de sepsis neonatal precoz en recién nacidos de término en países desarrollados, mientras que Escherichia coli se ha conver- tido en uno de los microorganismos más frecuentemente aislados en prematuros, particularmente los de extremo bajo peso al nacer $(\mathrm{EBPN})^{4,7,8}$. No se conocen reportes actuales sobre la epidemiología de la sepsis neonatal precoz en Uruguay.

Streptococcus del grupo B forma parte de la microbiota del tracto gastrointestinal materno desde el cual coloniza el aparato genital de la mujer, por lo que el recién nacido puede adquirir la infección como consecuencia de la invasión del líquido amniótico luego de la rotura de las membranas ovulares, durante su paso por el canal del parto o por vía hematógena?.

La prevalencia de colonización asintomática por SGB en el tercer trimestre del embarazo varía entre 10 y $30 \%$ en países desarrollados ${ }^{10,11}$. En Uruguay, en un estudio realizado por Laufer y cols., en el año 2008, sobre una muestra representativa de 300 mujeres embarazadas en nuestra maternidad, se encontró una prevalencia de portación de SGB en el exudado recto-vaginal de $17 \%$, cifra similar a las documentadas en otros países de América del Sur ${ }^{12}$. En el estudio realizado por Rivas y cols., en un medio mutual y que incluyó un total de 242 mujeres, $25 \%$ de las mujeres embarazadas resultaron portadoras de SGB entre las 35 y 37 semanas siendo la muestra ano-rectal la de mayor rendimiento ${ }^{13}$. 
Aproximadamente $50 \%$ de los recién nacidos son colonizados al nacimiento y $1 \%$ desarrollarán sepsis o enfermedad invasora por SGB (EISGB) ${ }^{10}$.

En la década del 90, los Centros para el Control y la Prevención de Enfermedades (CDC) de Atlanta, GA, en conjunto con el Colegio Americano de Obstetricia y Ginecología (ACOG) y la Academia Americana de Pediatría (AAP) publicaron las primeras estrategias de prevención para reducir la transmisión vertical de SGB en mujeres embarazadas con factores de riesgo ${ }^{14-16}$. En el año 2002, los CDC recomendaron el cribado prenatal universal a las 35-37 semanas de gestación para optimizar la identificación de aquellas madres en riesgo y que deberían recibir quimioprofilaxis antimicrobiana intraparto (IAP $)^{17}$. Tras la implementación de estas estrategias de prevención, se logró disminuir la incidencia de EISGB de 1,7 a 0,34-0,37 casos cada 1.000 nacidos vivos en Estados Unidos de América (E.U.A.) en el año 2010 ${ }^{11}$. Sin embargo, la incidencia mundial de EISGB sigue siendo alta con morbimortalidad significativa ${ }^{18,19}$.

Entre los recién nacidos que desarrollan EISGB, $83 \%$ presentan bacteriemia sin foco, $9 \%$ neumonía y $7 \%$ meningitis ${ }^{20}$. En $90 \%$ de los casos la enfermedad es de evolución rápida, haciéndose evidente al nacer o en las primeras $12 \mathrm{~h}$ de vida ${ }^{10}$.

Los recién nacidos sobrevivientes, particularmente los de EBPN, presentan mayor riesgo de secuelas neurológicas incluyendo parálisis cerebral, menor puntaje en la escala de desarrollo infantil de Bayley II y alteraciones de la visión y del crecimiento ${ }^{21}$.

El objetivo de este estudio fue determinar la incidencia, características clínicas y mortalidad asociada de la sepsis neonatal precoz por SGB e identificar las fallas en la prevención por parte del equipo de salud con miras a desarrollar nuevos planes de acción.

\section{Métodos}

Se realizó un estudio descriptivo, retrospectivo, sobre la incidencia y características clínicas de sepsis neonatal precoz por SGB en recién nacidos del Centro Hospitalario Pereira Rossell (CHPR) de Uruguay, durante los años 2007 y 2015, a partir de una cohorte de 66.075 nacidos vivos.

Los casos se definieron a partir del aislamiento de SGB de un sitio normalmente estéril en las primeras $72 \mathrm{~h}$ de vida y se obtuvieron de los registros en la base de datos del laboratorio de bacteriología del hospital.

Para el aislamiento de SGB se siguieron los protocolos estándares de procesamiento de muestras clínicas ${ }^{22}$. En base a estos datos aportados por el laboratorio, se procedió a buscar las historias clínicas de los pacientes en el sistema informático de registro del hospital.

El estudio fue aprobado por el Comité de Ética del CHPR.
Se describieron las características de la población en función de: edad materna, número de gestaciones, control de embarazo, complicaciones durante la gestación incluyendo infecciones urinarias durante cualquier trimestre del embarazo, estado de colonización materna por SGB según el resultado del exudado recto-vaginal, tiempo de rotura de las membranas ovulares, indicación de quimioprofilaxis intraparto y realización de la misma y vía de nacimiento.

Los datos recolectados en relación al recién nacido incluyeron edad gestacional, peso al nacer, puntaje de Apgar, necesidad de maniobras de resucitación, gasometría de cordón, síntomas iniciales y momento de presentación, tratamiento antimicrobiano realizado, gravedad de la enfermedad en función de la necesidad de ventilación, utilización de óxido nítrico y surfactante, requerimiento de inotrópicos, presencia de convulsiones, alta hospitalaria o fallecimiento. Se consideró mortalidad asociada a sepsis si el fallecimiento ocurrió en la primera semana de vida o si se determinó clínicamente que ésta fue la causa desencadenante de muerte.

\section{Definiciones operativas}

Durante el período de estudio se implementaron las recomendaciones del CDC del año 2002 de cribado prenatal universal a las 35-37 semanas de gestación y de quimioprofilaxis antimicrobiana intraparto para prevenir la enfermedad de adquisición vertical por $\mathrm{SGB}^{17}$. Se consideró colonización materna por SGB la identificación del microorganismo en el hisopado recto-vaginal.

Se indicó quimioprofilaxis intraparto en los siguientes casos: antecedente de hijo anterior con enfermedad invasora por SGB; bacteriuria por SGB durante cualquier trimestre del embarazo actual; exudado recto-vaginal positivo para SGB, excepto en caso de cesárea electiva con membranas ovulares íntegras y sin inicio del trabajo de parto; situación desconocida respecto al SGB y una de las siguientes: trabajo de parto pretérmino (menor a 37 semanas), fiebre intraparto (mayor a $38^{\circ} \mathrm{C}$ ) o rotura de membranas prolongadas (mayor a $18 \mathrm{~h})^{23}$.

\section{Análisis estadístico}

Las variables cualitativas se presentaron como frecuencias absolutas y relativas. Las variables numéricas se expresaron como media y valores máximos y mínimos. Se utilizó el software Epi Info ${ }^{\mathrm{TM}}$ para el procesamiento de los datos.

\section{Resultados}

\section{Incidencia}

Se identificaron 15 casos de sepsis neonatal precoz por SGB siendo la incidencia global en el período de estudio de 0,23 por 1.000 recién nacidos vivos. En todos los casos, 
al menos una muestra de hemocultivo fue positiva para SGB. En nuestro centro no se realizaba durante dicho período la serotipificación de las cepas aisladas.

La Figura 1 muestra la incidencia global reportada en distintas latitudes comparada con la reportada en nuestro estudio $^{24-35}$. La Figura 2 muestra la variación de la incidencia anual en nuestro centro en comparación con E.U.A. y Reino Unido durante el período de estudio.

\section{Factores maternos y estado de colonización}

La edad mediana materna fue de 20 años (rango 16-35). La mayoría de las mujeres embarazadas eran primigestas y presentaron cinco o más controles prenatales.

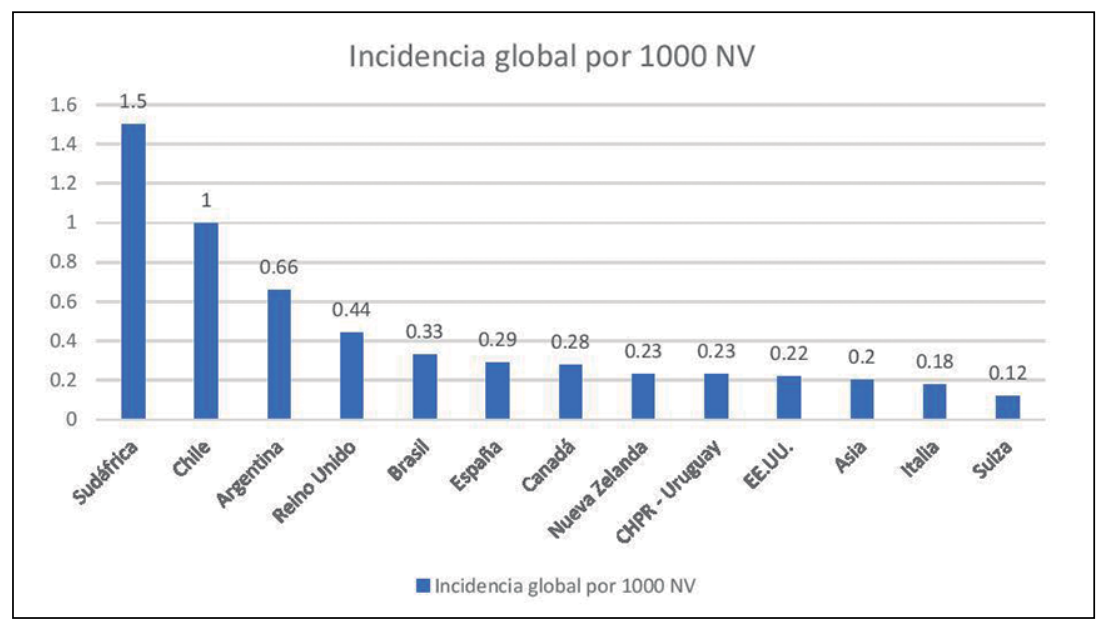

Figura 1. Incidencia de sepsis precoz por Streptococcus agalactiae en el período 2007-2015 a lo largo del mundo ${ }^{24-35}$. NV: nacidos vivos.

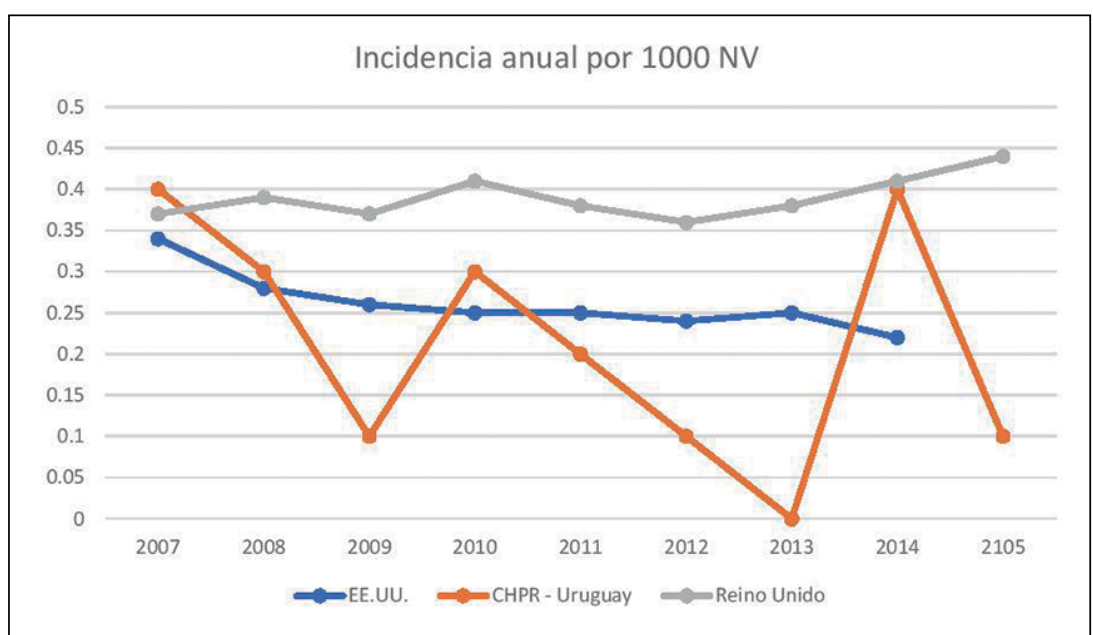

Figura 2. Incidencia anual de sepsis precoz por Streptococcus agalactiae. Comparación de nuestro centro CHPR con Estados Unidos de América y Reino Unido. Datos extraídos de: Active Bacterial Core Surveillance 2007 - 2014 (https://www.cdc.gov/abcs/reports-findings/surv-reports.html). Pyogenic and non-pyogenic streptococcal bacteraemia: annual data from voluntary surveillance (https://www.gov. uk/government/publications/pyogenic-and-non-pyogenic-streptococcal-bacteraemia-annual-data-fromvoluntary-surveillance).
De los tres casos de infección urinaria durante el embarazo, dos se debieron a $E$. coli y en un caso se desconoce el microorganismo causante. No se documentó infección urinaria causada por SGB en esta serie de casos.

El tiempo de rotura de membranas tuvo una mediana de $4 \mathrm{~h}$ (rango 0-24); un caso presentó una rotura prematura y prolongada de membranas de $24 \mathrm{~h}$ de evolución y en otro caso se desconoce el tiempo de rotura. Ninguna de las mujeres embarazadas presentó fiebre intraparto. En la Tabla 1 se detallan los datos obstétricos maternos.

En doce de las $15(80 \%)$ mujeres embarazadas no se obtuvo muestra recto-vaginal para búsqueda de SGB; en tres casos por tratarse de embarazos $<35$ semanas, en cuatro por tratarse de embarazos mal controlados en calidad y cantidad, y en cinco se desconoce el motivo de la omisión. De las tres (20\%) mujeres embarazadas que se realizó la pesquisa recto-vaginal, en una el cultivo fue positivo, uno negativo para SGB y en el otro caso no se encontraba disponible el resultado al momento del parto.

\section{Quimioprofilaxis antimicrobiana intraparto}

Cuatro de 15 (27\%) mujeres embarazadas presentaban indicación de profilaxis según los criterios del CDC 2002; en tres casos por trabajo de parto prematuro y en uno por colonización materna por SGB. De las mujeres embarazadas que no cumplían los criterios de profilaxis, 10 correspondieron a estado de colonización materna por SGB desconocido, sin factores de riesgo y uno por cribado negativo para SGB. No se realizó profilaxis intraparto en los cuatro casos en los que sí estaba indicada; en uno de ellos no alcanzó el tiempo de administrar la profilaxis durante el trabajo de parto. Se desconoce el motivo de la omisión en los otros tres casos.

\section{Datos del recién nacido}

La vía de nacimiento fue por parto vaginal en 11 casos $(73 \%)$ y por cesárea en 4 (27\%). La indicación de nacimiento por cesárea en todos los casos fue por trabajo de parto detenido. La mediana del peso al nacimiento fue de 3.045 g (rango 885-3.990); un recién nacido presentó un peso $<1.500 \mathrm{~g}$. La mediana de edad gestacional fue de 39 semanas (25-41); siendo tres recién nacidos $<37$ semanas. Tres de $15(20 \%)$ recién nacidos presentaron puntajes de Apgar < 7 al minuto de vida; uno de ellos (7\%) requirió resucitación en sala de parto con fármacos intra-traqueales correspondiendo a un prematuro de $885 \mathrm{~g}$. En la Tabla 2 se detallan los datos de los recién nacidos.

\section{Morbilidad y mortalidad neonatal asociada a sepsis por $S G B$}

En todos los casos, al menos una muestra de hemocultivo fue positiva para SGB. A su vez, de 10 cultivos extraídos de líquido cefalorraquídeo, uno resultó positivo para SGB (10\%). En tres recién nacidos no se realizó punción 


\begin{tabular}{|c|c|c|}
\hline \multicolumn{3}{|l|}{ Características } \\
\hline Edad materna (años) - mediana (rango) & 20 & $(16-35)$ \\
\hline Primigesta $-\mathrm{n}(\%)$ & 11 & (73) \\
\hline Controles prenatales $\geq 5-\mathrm{n}(\%)$ & 11 & (73) \\
\hline $\begin{array}{l}\text { Complicaciones durante el embarazo }-\mathrm{n}(\%)^{\dagger} \\
\text { Amenaza de parto prematuro } \\
\text { Infección urinaria } \\
\text { Rotura prematura y prolongada de membranas } \\
\text { Estado hipertensivo del embarazo } \\
\text { Diabetes mellitus gestacional } \\
\text { Restricción de crecimiento intrauterino } \\
\text { Infección genital baja }\end{array}$ & $\begin{array}{r}10 \\
4 \\
3 \\
1 \\
2 \\
1 \\
2 \\
3\end{array}$ & $\begin{array}{l}(67) \\
(40) \\
(30) \\
(10) \\
(20) \\
(10) \\
(20) \\
(30)\end{array}$ \\
\hline Realización de exudado recto-vaginal - n (\%) & 3 & (20) \\
\hline $\begin{array}{l}\text { Resultado de exudado recto-vaginal - } \mathrm{n}(\%) \\
\text { Positivo } \\
\text { Negativo } \\
\text { Pendiente al parto }\end{array}$ & $\begin{array}{l}1 \\
1 \\
1\end{array}$ & $\begin{array}{l}(33) \\
(33) \\
(33)\end{array}$ \\
\hline $\begin{array}{l}\text { Rotura de membranas* } \\
\text { Horas - mediana (rango) }\end{array}$ & 4 & $(0-24)$ \\
\hline Fiebre intraparto $>38^{\circ} \mathrm{C}$ & 0 & (0) \\
\hline Trabajo de parto prematuro & 3 & (20) \\
\hline $\begin{array}{l}\text { Indicación de quimioprofilaxis - n (\%) } \\
\text { Por prematurez } \\
\text { Exudado positivo a EGB }\end{array}$ & $\begin{array}{l}4 \\
3 \\
1\end{array}$ & $\begin{array}{l}(27) \\
(75) \\
(25)\end{array}$ \\
\hline Realización de quimioprofilaxis intraparto - n (\%) & 0 & (0) \\
\hline $\begin{array}{l}\text { Vía de nacimiento - n (\%) } \\
\text { Cesárea } \\
\text { Parto vaginal }\end{array}$ & $\begin{array}{r}4 \\
11\end{array}$ & $\begin{array}{l}(27) \\
(73)\end{array}$ \\
\hline
\end{tabular}

†Más de un diagnóstico por madre. *Datos perdidos: rotura de membranas (1 paciente, $n=14$ ).

lumbar por gravedad y en dos se desconoce el motivo de la omisión. En un recién nacido se aisló SGB en el cultivo de una lesión de piel.

Todos los casos se presentaron con sintomatología con una mediana de dos horas (rango 0-15). Doce recién nacidos presentaron inicialmente sintomatología respiratoria $(80 \%)$, tres presentaron hipotonía, hiporeactividad o dificultad en la succión $(20 \%)$, tres presentaron inestabilidad térmica (20\%), dos presentaron signos de intolerancia digestiva (13\%), uno se presentó con hipoglicemia que requirió corrección con aporte intravenoso $(7 \%)$, uno con shock séptico $(7 \%)$, y otro recién nacido presentó lesiones cutáneas ampollares generalizadas (7\%).

Siete recién nacidos $(47 \%)$ requirieron asistencia ventilatoria mecánica, de quienes dos (28\%) fueron por un período mayor a $48 \mathrm{~h}$.
Tabla 2. Características neonatales en pacientes con sepsis precoz por Streptococcus agalactiae $(n=15)^{*}$

\begin{tabular}{lcc}
\hline $\begin{array}{l}\text { Edad gestacional (semanas) - mediana (rango) } \\
\text { Menores de } 37 \text { semanas }-\mathrm{n}(\%)\end{array}$ & 39 & $(25-41)$ \\
Peso al nacer (gramos) - mediana (rango) & 3 & $(20)$ \\
BPN $<1.500 \mathrm{~g}-\mathrm{n}(\%)$ & 3.045 & $(885-3.990)$ \\
$\begin{array}{l}\text { Puntaje de Apgar }-\mathrm{n}(\%) \\
\quad<7 \text { al minuto de vida } \\
<7 \text { a los } 5 \text { min }\end{array}$ & 1 & $(7)$ \\
Necesidad de IOT* en sala de parto $-\mathrm{n}(\%)$ & 3 & $(20)$ \\
Resucitación completa en sala de parto $-\mathrm{n}(\%)$ & 2 & $(13)$ \\
Acidosis de cordón $-\mathrm{n}(\%)^{*}$ & 2 & $(13)$ \\
\hline
\end{tabular}

*Pérdida de datos: gasometría de cordón (5 pacientes, $\mathrm{n}=10$ ). IOT: intubación oro-traqueal.

\section{Tabla 3. Morbilidad y mortalidad asociada a sepsis precoz por Streptococcus agalactiae}

Sintomatología $-\mathrm{n}(\%)^{\dagger}$

Dificultad respiratoria

Shock

Lesiones en piel

Intolerancia digestiva

Hipoglicemia

Inestabilidad térmica

Dificultad en succión, hipotonía, hipo-reactividad

\begin{tabular}{|c|c|}
\hline 12 & (80) \\
\hline 1 & (7) \\
\hline 1 & (7) \\
\hline 2 & (13) \\
\hline 1 & (7) \\
\hline 3 & (20) \\
\hline 3 & (20) \\
\hline 2 & $(0-15)$ \\
\hline 7 & (47) \\
\hline 2 & (28) \\
\hline 8 & (53) \\
\hline 2 & (13) \\
\hline 3 & $(20)$ \\
\hline 15 & (100) \\
\hline 10 & $(67)$ \\
\hline 3 & $(20)$ \\
\hline 1 & (7) \\
\hline 1 & (7) \\
\hline 8 & (53) \\
\hline 2 & (13) \\
\hline 1 & $(7)$ \\
\hline 3 & (20) \\
\hline 2 & (13) \\
\hline 3 & (20) \\
\hline
\end{tabular}

Inicio de la sintomatología (horas) - mediana (rango)

Ventilación mecánica - n (\%)

$>48 \mathrm{~h}$

Fármacos inotrópicos - n (\%)

Óxido nítrico inhalado - $\mathrm{n}(\%)$

Surfactante - n (\%)

Forma de presentación - n (\%)

Bacteriemia

Bacteriemia sin foco evidente

Neumonía

Meningitis

Infección de piel

Complicaciones durante la hospitalización - n (\%) ${ }^{\dagger}$

Shock séptico o inestabilidad hemodinámica

Hipertensión pulmonar

Apnea

Convulsiones

Daño multiorgánico

$(0-20)$

Días de hospitalización entre sobrevivientes - media (rango**

Fallecimientos - n (\%)

†Más de un diagnóstico por recién nacido. *Pérdida de datos: días de antimicrobianos ( 2 pacientes, $n=13$ ), días de internación entre pacientes vivos (5 pacientes, $\mathrm{n}=10$ ). 
Entre las complicaciones en la evolución se destaca: shock séptico o signos de inestabilidad hemodinámica con necesidad de fármacos inotrópicos $(53 \%)$, convulsiones (20\%), hipertensión pulmonar que requirió tratamiento con óxido nítrico inhalado (13\%), falla multi-orgánica (13\%) y apnea (7\%). Tres recién nacidos no presentaron complicación durante la hospitalización (20\%).

Fallecieron tres $(20 \%)$ neonatos con sepsis precoz por SGB mientras que los demás evolucionaron favorablemente. De los fallecidos, dos correspondieron a prematuros de 25 y 32 semanas de gestación cuyo desencadenante de muerte fue neumonía con shock séptico y el tercero correspondió a un recién nacido de 40 semanas de gestación que se presentó con shock séptico y falla multi-orgánica. Todos los decesos ocurrieron en las primeras $24 \mathrm{~h}$ de vida (Tabla 3 ).

\section{Antibioterapia neonatal}

La mayoría de los recién nacidos (93\%) recibió ampicilina y gentamicina frente al diagnóstico presuntivo de sepsis neonatal precoz. Una vez recibidos los resultados de los cultivos, no se modificaron los regímenes antimicrobianos, incluso para el caso con meningitis. En el recién nacido con lesiones en piel se indicó ampicilina asociado a cefuroxima ev.

\section{Discusión}

La implementación del cribado universal para la búsqueda de SGB entre las 35 y 37 semanas de edad gestacional y de la quimioprofilaxis intraparto ha impactado considerablemente sobre la incidencia de sepsis neonatal precoz por SGB con cifras reportadas de hasta $80 \%$ para E.U.A. ${ }^{23}$. La incidencia de sepsis precoz por SGB en nuestro centro se ha mantenido estable sin grandes variaciones por debajo de 0,40 cada 1.000 recién nacidos vivos, con una incidencia global en los nueve años de estudio de 0,23 por 1.000 recién nacidos vivos, similar a las cifras reportadas en otros centros donde se implementa quimioprofilaxis intraparto ${ }^{29-32}$. Sin embargo, no contamos con datos anteriores a la era de la quimioprofilaxis en nuestro centro que nos permita comparar la variación de incidencia de sepsis precoz a SGB en ambos períodos.

El cribado prenatal de portación de SGB no fue realizado en 12 de las 15 mujeres embarazadas. El motivo de esta omisión, en general se corresponde con una falla en la adherencia a las estrategias de prevención, tanto por parte del equipo de salud como de la población en cuestión. Identificar de forma oportuna y eficaz a aquellas mujeres portadoras de SGB al momento del parto es crucial para implementar la quimioprofilaxis intraparto y de esta manera reducir el riesgo de sepsis.

En este escenario, sería beneficioso contar con métodos diagnósticos basados en la identificación de SGB en exudado recto-vaginal mediante técnica de reacción de polimerasa en cadena (RPC) en el momento del parto, método rápido que permite tener los resultados en aproximadamente una hora, con un buen desempeño diagnóstico en comparación con los métodos estándares de cultivo utilizados en la actualidad ${ }^{36-40}$. Asimismo, no se realizó quimioprofilaxis intraparto en nuestra población de estudio. En la mayoría de estos casos, esta omisión se debió a que la consulta se realizaba en fases avanzadas del trabajo de parto $y$, en menor medida, por una pobre adherencia al protocolo al momento del parto.

Doce de las mujeres embarazadas de este estudio no tenían indicación de quimioprofilaxis de acuerdo al protocolo del $\mathrm{CDC}^{23}$, ya que, si bien el estado de colonización por SGB era desconocido, no presentaban factores de riesgo adicionales. Esto resalta la importancia de conocer el estado de colonización materno por SGB al momento del parto para guiar el manejo perinatal. Sin embargo, se debe destacar que el hallazgo de un cultivo negativo para SGB no debe descartar la presencia de sepsis en el recién nacido cuando la clínica es sugestiva, como ocurrió en nuestro estudio. La colonización materna con posterioridad a la realización del muestreo, el modo de recolección de la muestra, su conservación y procesamiento, la presencia de cepas de SGB no hemolíticas (aproximadamente 2\%), uso de antimicrobianos y problemas en el registro de los resultados pueden ser causas de resultados falsamente negativos ${ }^{4,23,41}$.

Todos los recién nacidos presentaron sintomatología de inicio precoz dentro de las primeras $15 \mathrm{~h}$ de vida, siendo el cuadro predominante la dificultad respiratoria. Si bien tres recién nacidos presentaron alteraciones en la esfera neurológica, solamente se constató un caso de meningitis (7\%). A partir de datos epidemiológicos en relación a la forma de presentación de la EISGB en E.U.A., se observó una incidencia menor de meningitis asociada a sepsis precoz (7\%) en comparación con la sepsis tardía $(27 \%)$, lo que es consistente con nuestros hallazgos ${ }^{20}$. Si bien la mitad de los casos presentaron shock o inestabilidad hemodinámica que requirieron apoyo inotrópico y ventilación mecánica, la mayoría evolucionó de forma favorable en el corto plazo. No contamos con datos sobre el seguimiento a largo plazo.

Con los avances en los cuidados neonatales y las estrategias de prevención de sepsis neonatal, la tasa de mortalidad ha disminuido considerablemente desde aproximadamente $50 \%$ en la década del 70 hasta 4 a $6 \%$ en la actualidad. Sin embargo, la mortalidad continúa siendo elevada para los recién nacidos prematuros, particularmente bajo 33 semanas de gestación, en quienes alcanza cifras de $30 \%{ }^{23}$. En nuestro centro se registró una tasa de mortalidad de $20 \%$ asociada a sepsis neonatal precoz para SGB, correspondiendo a la mayoría de los casos a recién nacidos prematuros. 
La prevención de la sepsis neonatal comienza con la prevención del parto prematuro. Por esto, debemos dirigir nuestros esfuerzos en colaborar como parte de un equipo multidisciplinario asegurando el cumplimiento de las estrategias de prevención. Es necesario incrementar la búsqueda prenatal de SGB mediante el fortalecimiento de las metas asistenciales y de la adherencia a los protocolos de atención en el control prenatal en nuestra población, así como mejorar el rendimiento de las técnicas diagnósticas utilizadas al momento del parto y lograr la tipificación de las cepas aisladas, con miras a optimizar las acciones terapéuticas.

El carácter retrospectivo es la principal limitación de este estudio.

\section{Conclusión}

Este estudio es el primer reporte sobre la incidencia y morbimortalidad de sepsis neonatal precoz a SGB en Uruguay. La incidencia de sepsis precoz por SGB en el CHPR entre 2007 y 2015 fue de 0,23 por 1.000 recién nacidos vivos, similar a la incidencia en centros donde se realiza quimioprofilaxis. Todos los casos presentaron sintomatología en el primer día de vida, siendo la presentación clínica más frecuente la dificultad respiratoria.

El conocimiento local de la epidemiología y su forma de presentación es fundamental para diseñar estrategias que, en conjunto, contribuyan a un descenso significativo de la incidencia, morbilidad y mortalidad, y que a su vez permitan optimizar recursos y disminuir los costos de salud asociados a esta enfermedad.
Agradecimientos: Al Profesor Daniel Borbonet y al Profesor José L. Tapia por su colaboración en la preparación del material.

\section{Resumen}

Introducción. La infección por Streptococcus agalactiae ( $\beta$-hemolítico del grupo B (SGB) continúa siendo una de las principales causas de sepsis precoz en países desarrollados a pesar de la implementación de profilaxis efectiva. Objetivos Describir la incidencia, características clínicas y mortalidad de sepsis precoz por SGB en recién nacidos del Centro Hospitalario Pereira Rossell (CHPR), y analizar las fallas de adherencia a las estrategias de prevención. Métodos. Estudio retrospectivo de descripción de casos entre los años 2007 a 2015 identificados a partir de la base de datos del laboratorio de bacteriología. Resultados. Se identificaron 15 casos de sepsis neonatal precoz a SGB con una incidencia en el período de estudio de $0,23 \%$. La quimioprofilaxis intraparto no fue realizada en caso alguno. Todos los recién nacidos se presentaron sintomáticos en las primeras 15 h de vida. La dificultad respiratoria fue el signo más frecuente $(80 \%)$. En un caso se aisló SGB de líquido cefalorraquídeo. La mortalidad fue de $20 \%$. Todas las muertes ocurrieron en las primeras $24 \mathrm{~h}$ de vida, siendo dos tercios prematuros. Conclusión. La incidencia de sepsis precoz por SGB en el CHPR fue similar a la incidencia en centros donde se realiza quimioprofilaxis. Una mejor adherencia a las estrategias de prevención podría disminuir la incidencia.

\section{Referencias bibliográficas}

1.- Camacho-González A, Spearman P W, Stoll B J. Neonatal infectious diseases: evaluation of neonatal sepsis. Pediatr Clin North Am 2013; 60 (2): 367-89. doi: 10.1016/j.pcl.2012.12.0.

2.- Zea-Vera A, Ochoa T J. Challenges in the diagnosis and management of neonatal sepsis. J Trop Pediatr 2015; 61 (1): 1-13. doi: 10.1093/ tropej/fmu079.

3.- Zea-Vera A, Turin C G, Ochoa T J. Unifying criteria for late neonatal sepsis: proposal for an algorithm of diagnostic surveillance. Rev Peruana Med Exp Salud Pública 2014; 31 (2): 358-63.

4.- Stoll B J, Hansen N I, Sánchez P J, Faix R G, Poindexter B B, Van Meurs K P, et al. Early onset neonatal sepsis: the burden of group B streptococcal and $E$. coli disease continues. Pediatrics 2011; 127 (5): 817-26. doi: 10.1542/ peds 2010-2217.

5.- Shane A L, Stoll B J. Neonatal sepsis: progress towards improved outcomes. J Infect 2014; 68 Suppl 1: S24-32. doi: 10.1016/j. jinf.2013.09.011.

6.- Hornik C P, Fort P, Clark R H, Watt K, Benjamin D K, Smith P B, et al. Early and late onset sepsis in very-low-birth-weight infants from a large group of neonatal intensive care units. Early Human Develop 2012; 88 (Suppl 2): S69-S74. doi: 10.1016/S03783782(12)70019-1.

7.- Stoll B J, Hansen N, Fanaroff A A, Wright L L, Carlo W A, Ehrenkranz R A, et al. Changes in pathogens causing early-onset sepsis in very-low-birth-weight infants. New Engl J Med. 2002; 347(4): 240-7. DOI: 10.1056/ NEJMoa012657.

8.- Vergnano S, Menson E, Kennea N, Embleton N, Russell A B, Watts T, et al. Neonatal infections in England: the NeonIN surveillance network. Arch Dis Child Fetal Neonatal Ed 2011; 96 (1): F9-f14. doi: 10.1136/ adc.2009.178798.
9.- $\quad$ Edwards M S, Nizet V, Baker C J. Chapter 12 - Group B Streptococcal Infections. Infectious Diseases of the Fetus and Newborn (Seventh Edition). Philadelphia: W.B. Saunders; 2011. p. 419-69.

10.- Heath P T, Schuchat A. Perinatal group B streptococcal disease. Best Pract Res Clin Obstet Gynaecol. 2007; 21(3): 411-24. DOI: 10.1016/j.bpobgyn.2007.01.003.

11.- Verani J R, McGee L, Schrag S J. Prevention of perinatal group B streptococcal disease-revised guidelines from CDC, 2010. MMWR Recommendations and reports: Morb Mortal Wkly Report Recommendations and Reports / Centers for Disease Control. 2010; 59(Rr-10): $1-36$.

12.- Laufer J, Scasso S, Sosa C G, Rodríguez-Cuns G, Alonso J, Pons J E. Group B streptococcus colonization among pregnant women in Uruguay. Intern J Gynaecol Obstet 2009; 104 (3): 242-3. doi: 10.1016/j.ijgo.2008.10.020.

13.- Rivas C, Tallac I, Etchenique A. Colonización 
vaginorrectal por Streptococcus del grupo B en mujeres embarazadas, entre las 35 a 37 semanas de gestación. Rev Méd Uruguay 2006; 22: 1916 .

14.- Prevention of perinatal group B streptococcal disease: a public health perspective. Centers for Disease Control and Prevention. MMWR Recommendations and Reports : Morbid Mortal Wkly Rep Recommendations Reports / Centers for Disease Control 1996; 45 (Rr-7): 1-24.

15.- ACOG committee opinion. Prevention of early-onset group B streptococcal disease in newborns. Number 173-June 1996. Committee on Obstetric Practice. American College of Obstetrics and Gynecologists. Intern J Gynaecol Obstet 1996; 54 (2): 197-205.

16.- Revised guidelines for prevention of earlyonset group B streptococcal (GBS) infection. American Academy of Pediatrics Committee on Infectious Diseases and Committee on Fetus and Newborn. Pediatrics 1997; 99 (3): 489-96.

17.- Schrag S, Gorwitz R, Fultz-Butts K, Schuchat A. Prevention of perinatal group B streptococcal disease. Revised guidelines from CDC. MMWR Recommendations Rep: Morbid Mortal Wkly Rep Recommendations Rep / Centers for Disease Control 2002; 51 (Rr-11): 1-22.

18.- Edmond K M, Kortsalioudaki C, Scott S, Schrag S J, Zaidi A K, Cousens S, et al. Group $\mathrm{B}$ streptococcal disease in infants aged younger than 3 months: systematic review and metaanalysis. Lancet (London, England) 2012; 379 (9815): 547-56. DOI:10.1016/S01406736(11)61651-6.

19.- Melin P. Neonatal group B streptococcal disease: from pathogenesis to preventive strategies. Clin Microbiol Infect 2011; 17 (9): 1294-303. doi: 10.1111/j.14690691.2011.03576.x.

20.- Phares C R, Lynfield R, Farley M M, Mohle-Boetani J, Harrison L H, Petit S, et al. Epidemiology of invasive group B streptococcal disease in the United States, 1999-2005. JAMA 2008; 299 (17): 2056-65. doi: 10.1001/jama.299.17.2056.

21.- Stoll B J, Hansen N I, Adams-Chapman I, Fanaroff A A, Hintz S R, Vohr B, et al. Neurodevelopmental and growth impairment among extremely low-birth-weight infants with neonatal infection. JAMA 2004; 292 (19): 2357-65. DOI: 10.1001/jama.292.19.2357.

22.- Jorgensen J H, Carroll K C. Manual of Clinical Microbiology, 11th ed.: ASM Press Washington, D.C.; 2015.

23.- Recommendations for the Prevention of perinatal group b streptococcal (GBS) disease. Pediatrics 2011; 128 (3): 611. doi: 10.1542/ peds.2011-1466.

24.- Larcher J S, Cappellino F, Giusto R D, Travella C. Group B streptococcus colonization during pregnancy and prevention of early onset of disease. Medicina (Buenos Aires) 2005; 65 (3): 201-6.

25.- Tapia I J L, Reichhard T C, Saldías R M I, Abarzúa C F, Pérez A M E, González M Á, et al. Sepsis neonatal en la era de profilaxis antimicrobiana prenatal. Rev Chilena Infectol 2007; 24: 111-6. http://dx.doi.org/10.4067/ S0716-10182007000200004.

26.- Freitas F T dM, Romero G A S. Early-onset neonatal sepsis and the implementation of group B streptococcus prophylaxis in a Brazilian maternity hospital: a descriptive study. Braz J Infect Dis 2017; 21 (1): 92-7. doi: 10.1016/j.bjid.2016.09.013.

27.- Berardi A, Baroni L, Bacchi Reggiani M L, Ambretti S, Biasucci G, Bolognesi S, et al. The burden of early-onset sepsis in Emilia-Romagna (Italy): a 4-year, population-based study. J Matern Fetal Neonatal Med 2016; 29 (19): 3126-31. doi: 10.3109/14767058.2015.1114093.

28.- Giannoni E, Berger C, Stocker M, Agyeman P, Posfay-Barbe K M, Heininger U, et al. Incidence and outcome of group B streptococcal sepsis in infants in Switzerland. Pediatr Infect Dis J 2016; 35 (2): 222-4. doi: 10.1097/INF.0000000000000974.

29.- Gimenez M, Sanfeliu I, Sierra M, Dopico E, Juncosa T, Andreu A, et al. Group B streptococcal early-onset neonatal sepsis in the area of Barcelona (2004-2010). Analysis of missed opportunities for prevention]. Enf Infec Microbiol Clin 2015; 33 (7): 446-50. doi: 10.1016/j.eimc.2014.10.015.

30.- Schrag S J, Farley M M, Petit S, Reingold A, Weston E J, Pondo T, et al. Epidemiology of invasive early-onset neonatal sepsis, 2005 to 2014. Pediatrics 2016; 138 (6). DOI: 10.1542/ peds.2016-2013.

31.- Poliquin V, Cohen E, Poliquin P G, Schneider C, Menticoglou S. Ongoing cases of early-onset group B streptococcal disease in the era of screening and prophylaxis. J Obstet Gynaecol Can 38 (10): 926-9. doi: 10.1016/j.jogc.2016.05.006.

32.- Darlow B A, Voss L, Lennon D R, Grimwood $\mathrm{K}$. Early-onset neonatal group B streptococcus sepsis following national risk-based prevention guidelines. Aust N Z J Obstet Gynaecol 2016; 56 (1): 69-74. doi: 10.1111/ajo.12378.
33.- Quan V, Verani J R, Cohen C, von Gottberg A, Meiring S, Cutland C L, et al. Invasive group B streptococcal disease in South Africa: importance of surveillance methodology. PloS one 2016; 11 (4): [e0152524 https://doi. org/10.1371/journal.pone.0152524.

34.- Villanueva-Uy M E, Wongsiridej P, Sangtawesin V, Chiu V, Tallo V, NazaireBermal N, et al. The burden of invasive neonatal group B streptococcal (GBS) disease in Thailand and the Philippines. Southeast Asian J Trop Med Public Health 2015; 46 (4): 728-37.

35.- Pyogenic and non-pyogenic streptococcal bacteraemia: annual data from voluntary surveillance (https://www.gov.uk/government/ publications/pyogenic-and-non-pyogenicstreptococcal-bacteraemia-annual-data-fromvoluntary-surveillance) [cited 2016].

36.- de Tejada B M, Pfister R E, Renzi G, Francois $\mathrm{P}$, Irion $\mathrm{O}$, Boulvain $\mathrm{M}$, et al. Intrapartum group B streptococcus detection by rapid polymerase chain reaction assay for the prevention of neonatal sepsis. Clin Microbiol Infect 2011; 17 (12): 1786-91. doi: 10.1111/j.14690691.2010.03378.x.

37.- Rallu F, Barriga P, Scrivo C, Martel-Laferriere $\mathrm{V}$, Laferriere C. Sensitivities of antigen detection and PCR assays greatly increased compared to that of the standard culture method for screening for group B streptococcus carriage in pregnant women. J Clin Microbiol 2006; 44 (3): 725-8. DOI: $10.1128 /$ JCM.44.3.725-728.2006.

38.- El Helali N, Nguyen J C, Ly A, Giovangrandi Y, Trinquart L. Diagnostic accuracy of a rapid real-time polymerase chain reaction assay for universal intrapartum group B streptococcus screening. Clin Infect Dis 2009; 49 (3): 417-23. doi: 10.1086/600303.

39.- Park J S, Cho D H, Yang J H, Kim M Y, Shin S M, Kim E C, et al. Usefulness of a rapid real-time PCR assay in prenatal screening for group B streptococcus colonization. Ann Lab Med 2013; 33 (1): 39-44. doi: 10.3343/ alm.2013.33.1.39.

40.- Ke D, Bergeron M G. Molecular methods for rapid detection of group B streptococci. Expert Rev Mol Diagn 2001;1 (2): 175-81. DOI: 10.1586/14737159.1.2.175.

41.- Van Dyke M K, Phares C R, Lynfield R, Thomas A R, Arnold K E, Craig A S, et al. Evaluation of universal antenatal screening for group B streptococcus. N Engl J Med. 2009; 360 (25): 2626-36. doi: 10.1056/ NEJMoa0806820. 